\title{
BMJ
}

\section{Cost effectiveness of pneumococcal vaccination among Dutch infants: an economic analysis of the seven valent pneumococcal conjugated vaccine and forecast for the 10 valent and 13 valent vaccines}

'Unit of PharmacoEpidemiology and PharmacoEconomics,

Department of Pharmacy,

University of Groningen, Groningen, Netherlands

${ }^{2}$ Wilhelmina Children's Hospital, University Medical Center Utrecht, Utrecht, Netherlands

${ }_{3}^{3}$ Julius Center for Health Sciences and Primary Care, University of Utrecht, Utrecht, Netherlands

${ }^{4}$ Center for Infection and Immunity Amsterdam,

Department of Medical Microbiology and the Netherlands Reference Laboratory for Bacterial Meningitis, Academic Medical

Center Amsterdam, Amsterdam,

Netherlands

${ }^{5}$ Netherlands Vaccine Institute, Bilthoven, Netherlands

${ }^{6}$ Department of Epidemiology, University Medical Center Groningen, University of

Groningen, Groningen, Netherlands

Correspondence to: $\mathrm{M} \mathrm{H}$

Rozenbaum

m.h.rozenbaum@rug.nt

Cite this as: BMJ 2010;340:C2509 doi:10.1136/bmi.c2509

\begin{abstract}
Mark H Rozenbaum, health economist and modeller of infectious diseases, ${ }^{1}$ Elisabeth AM Sanders, professor in paediatric immunology and infectious diseases, ${ }^{2}$ Albert Jan van Hoek, health economist and modeller of infectious diseases, ${ }^{1}$ Angelique G S C Jansen, research fellow, ${ }^{2,3}$ Arie van der Ende, associate professor medical microbiology, ${ }^{4}$ Germie van den Dobbelsteen, senior research scientist, ${ }^{5}$ Gerwin D Rodenburg, research fellow, ${ }^{2}$ Eelko Hak, associate professor in clinical epidemiology of infectious diseases, ${ }^{1,23,6}$ Maarten J Postma, professor in pharmacoeconomics ${ }^{1,6}$
\end{abstract}

\section{ABSTRACT}

Objectives To update cost effectiveness estimates for the four dose $(3+1)$ schedule of the seven valent pneumococcal conjugated vaccine (PCV-7) in the Netherlands and to explore the impact on cost effectiveness of reduced dose schedules and implementation of 10 valent and 13 valent pneumococcal vaccines (PCV-10 and PCV-13).

Design Economic evaluation comparing PCV-7, PCV-10, and PCV-13 with no vaccination using a decision tree analytic model built from data in previous studies.

Setting The Netherlands.

Population A cohort of 180000 newborns followed until 5 years of age.

Main outcome measures Costs; gains in life years and quality adjusted life years (QALYs); and incremental cost effectiveness ratios.

Results Under base case assumptions-that is, assuming a five year protective period of the vaccine and no assumed net indirect effects (herd protection minus serotype replacement) among children aged over 5 years -vaccination with PVC-7 in a four dose $(3+1)$ schedule was estimated to prevent 71 and 5778 cases of invasive and non-invasive pneumococcal disease, respectively, in children aged up to 5 years. This corresponds with a total net gain of 173 life years or 277 QALYs. The incremental cost effectiveness ratio of PCV-7 was estimated at $€ 113891$ ( 988300 ; \$145 000) per QALY, well over the ratio of $€ 50000$ per QALY required for PCV-7 to be regarded as potentially cost effective. A three dose $(2+1)$ schedule of PCV-7 reduced the incremental cost effectiveness ratio to $€ 82975$ per QALY. For various assumptions and including $10 \%$ of the maximum net indirect effects among individuals aged 5 years and over, PCV-10 and PCV-13 had incremental cost effectiveness ratios ranging from $€ 31250$ to $€ 52947$ per QALY.
Conclusions The current Dutch infant vaccination programme of four doses of PCV-7 is not cost effective because of increases in invasive disease caused by nonvaccine serotypes, which reduces the overall direct effects of vaccination and offsets potential positive herd protection benefits in unvaccinated individuals. The 10 valent and 13 valent pneumococcal vaccines could have better net health benefits than PCV-7 through less replacement disease and increased herd protection. Both these effects could substantially reduce the incremental cost effectiveness ratio to possibly acceptable levels, if total programme costs can be lowered by reduced schedules, reductions in vaccine prices, or both.

\section{INTRODUCTION}

Given the multitude of new vaccines available for introduction into national immunisation programmes, health economic modelling of various immunisation plans is becoming increasingly important in informing decisions on health policy. The decision to introduce the seven valent pneumococcal conjugated vaccine (PCV-7) into the Dutch national immunisation programme for infants, for example, has in part been driven by cost effectiveness considerations. ${ }^{1}$ The Dutch Health Council estimated the incremental cost effectiveness ratio of vaccination with $\mathrm{PCV}-7$ compared with no vaccination at $€ 70000$ ( $£ 60300 ; \$ 89200)$ and less than $€ 20000$ per quality adjusted life year (QALY) in 2001 and 2005, respectively. ${ }^{1}$ Crucial factors responsible for the change from a potentially unfavourable cost effectiveness ratio in 2001, exceeding $€ 50000$ per QALY, to a favourable ratio in 2005 were the inclusion of data on observed herd protection effects in adults after nationwide implementation of $\mathrm{PCV}-7$ in the USA in 2000 and limited disease development caused by pneumococcal serotypes not present in the 


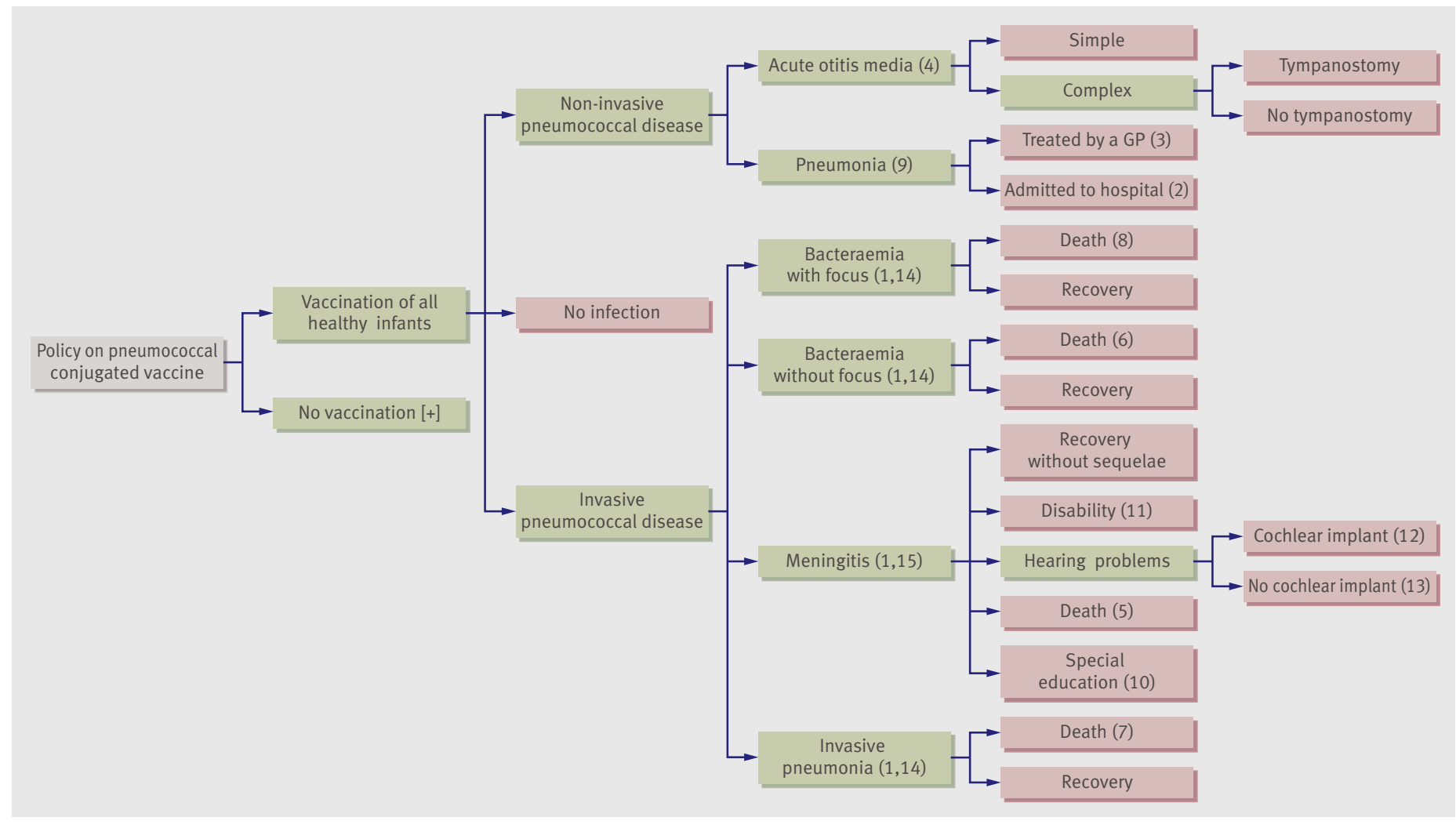

Fig 1|Decision tree used in conjunction with the cohort of 180000 newborns. Numbers between brackets correspond to data shown in table 1 . The boxes represent decision nodes, with green colour indicating probabilistic states and red colour indicating end states. The "No vaccination" arm is a clone of the "Vaccination of all healthy infants" arm (as represented by the + sign; risks differ between both arms)

PCV-7 replacing pneumococcal serotypes eliminated by the vaccine (replacement disease)..$^{-4}$

Next to direct effects on invasive disease in vaccinees, expected savings from herd protection were also part of health economic studies in other European countries that introduced PCV-7 into their national immunisation programmes. ${ }^{5-10}$ Both the four dose $(3+1)$ vaccine schedule and the reduced three dose $(2+1)$ schedule, as implemented in Norway and the UK, ${ }^{112}$ are highly effective against invasive pneumococcal disease caused by vaccine serotypes. However, the net overall benefit of national immunisation programmes in many European countries has been reduced by increases in invasive disease caused by non-vaccine serotypes. ${ }^{12-15}$ Importantly, in the first 18-30 months after the introduction of PCV7 in the Netherlands, France, and the UK, no overall reduction in invasive disease in non-vaccinees was observed. ${ }^{121315}$

Given that both increases in invasive disease caused by non-vaccine serotypes and absence of herd protection may considerably affect the cost effectiveness of the current Dutch vaccination programme, we set out to update cost effectiveness estimates for the current four dose schedule of PCV-7 by using recent data on epidemiology and resource use. Also, we investigate the cost effectiveness of reduced dose schedules and vaccine price reductions combined with the implementation of 10 valent and 13 valent pneumococcal vaccines (PCV-10 and PCV-13).

\section{METHODS}

Model

We designed a decision tree analytic model structure that builds on our previously reported model ${ }^{6}{ }^{6}$ Various data sources were used to populate our model; these included clinical trials and observational studies for effectiveness of pneumococcal vaccines, laboratory data for incidence and serotype distributions of pneumococcal disease, and registrations for resource use and costs. Figure 1 shows the disease model for the health effects of pneumococcal vaccination, including the possibility of subsequent pneumococcal disease such as non-invasive pneumonia, otitis media, and invasive pneumococcal disease. Assumptions regarding both costs and quality of life are summarised in table 1 and are more thoroughly discussed in web extra 1 .

In the analyses, a cohort of 180000 newborns, representing the Dutch birth cohort, was run through the decision tree twice (base case analysis): once as a mainly vaccinated cohort (PCV-7/PCV-10/PCV-13); and once as an unvaccinated cohort. The analytic time frame of the study was five years because vaccine effectiveness could not be assumed beyond five years. However, long term effects of invasive pneumococcal disease were extrapolated over the full lifetime of the individuals in the cohort (that is, until death or 100 years). 
Table 1|Parameters used in the economic model

\begin{tabular}{|c|c|c|c|c|}
\hline & Mean or range & Distribution & $\begin{array}{l}\text { Corresponding } \\
\text { branches in fig } 1\end{array}$ & References \\
\hline \multicolumn{5}{|l|}{ Case-fatality rate (birth cohort) } \\
\hline Meningitis & $9 \%$ & Beta $(3,32)$ & 5 & 17 \\
\hline Pneumonia & $0 \%$ & $\mathrm{~N} / \mathrm{A}$ & $\mathrm{N} / \mathrm{A}$ & 17 \\
\hline Bacteraemia with focus & $0 \%$ & $\mathrm{~N} / \mathrm{A}$ & N/A & 17 \\
\hline Bacteraemia without focus & $9 \%$ & Beta $(2,21)$ & 6 & 17 \\
\hline Mortality (non-invasive pneumonia and acute otitis media) & $0 \%$ & $\mathrm{~N} / \mathrm{A}$ & $\mathrm{N} / \mathrm{A}$ & Assumed \\
\hline \multicolumn{5}{|l|}{ Case-fatality rate (age five years or older) } \\
\hline Meningitis & $9-92 \%$ & Beta (age dependent) & 5 & 17 \\
\hline Pneumonia & $0-29 \%$ & Beta (age dependent) & 7 & 17 \\
\hline Bacteraemia with focus & $0-33 \%$ & Beta (age dependent) & 8 & 17 \\
\hline Bacteraemia without focus & $9-67 \%$ & Beta (age dependent) & 6 & 17 \\
\hline Respiratory infections & $0 \%$ & $\mathrm{~N} / \mathrm{A}$ & $\mathrm{N} / \mathrm{A}$ & Assumed \\
\hline \multicolumn{5}{|l|}{ Vaccine efficacy } \\
\hline Invasive pneumococcal disease (all vaccine serotypes) & $97.4 \%$ & Log normal (SE 0.044) & 1 & 19 \\
\hline Non-invasive pneumonia (admitted to hospital) & $11.1 \%$ & Log normal (SE 0.082) & 2 & 23 \\
\hline Non-invasive pneumonia (seen by general practitioner) & $6.0 \%$ & Log normal (SE 0.032) & 3 & 23 \\
\hline Acute otitis media & $7.0 \%$ & Log normal (SE 0.011) & 4 & 22 \\
\hline \multicolumn{5}{|l|}{ Direct costs $(€)$} \\
\hline \multicolumn{5}{|l|}{ Cost of hospital admission* } \\
\hline Invasive pneumococcal disease (age dependent) & $1091-27318$ & Triangular (age dependent) & 1 & 17,39 \\
\hline Non-invasive pneumonia & $26-2614$ & Triangular (severity dependent) & 9 & $16,39,40$ \\
\hline Acute otitis media & $17-381$ & Triangular (severity dependent) & 4 & $16,39,40$ \\
\hline Special education (annual costs) & $9798-16962$ & Triangular (age dependent) & 10 & 16 \\
\hline Institutional care (annual costs) & 39583 & Triangular $(29,687 ; 39,583 ; 49,478)$ & 11 & 39 \\
\hline Cochlear implantation & 56633 & Triangular $(0 ; 0.004 ; 0.01)$ & 12 & 41 \\
\hline \multicolumn{5}{|l|}{ Indirect costs $(€)$} \\
\hline Invasive pneumococcal disease $†$ & $0-974$ & Triangular (severity dependent) & 1 & 17,39 \\
\hline Non-invasive pneumonia (admitted to hospital) $\ddagger$ & $0-2529$ & Triangular (severity dependent) & 1 & 17,39 \\
\hline Non-invasive pneumonia (seen by general practitioner)† & $115-315$ & Triangular (severity dependent) & 9 & 16,39 \\
\hline Acute otitis media† & $58-23$ & Triangular (severity dependent) & 4 & 16,39 \\
\hline \multicolumn{5}{|l|}{ Total drop in quality of life (QALYs) } \\
\hline Disability§ & 0.53 & Beta (estimated) & 11 & 42 \\
\hline Bilateral hearing loss (first year)§ & 0.45 & Beta (estimated) & 12 & 8,43 \\
\hline Bilateral hearing loss: cochlear device§ & 0.18 & Beta (estimated) & 12 & 8,43 \\
\hline All other hearing loss§ & 0.09 & Beta (estimated) & 13 & 42 \\
\hline Hospital admission for bacteraemia** & 0.0079 & Beta (estimated) & 14 & 8,44 \\
\hline Hospital admission for meningitis & 0.0232 & Beta (estimated) & 15 & 8,44 \\
\hline Hospital admission for non-invasive pneumoniaף & 0.006 & Triangular $(0.001,0.006,0.01)$ & 2 & 8 \\
\hline Non-invasive pneumonia treated by a general practitioner & 0.004 & Triangular $(0,0.004,0.01)$ & 3 & 8 \\
\hline Acute otitis mediaף & 0.005 & Triangular $(0,0.005,0.01)$ & 4 & 8 \\
\hline \multicolumn{5}{|l|}{ Other parameters } \\
\hline Increase in non-vaccine serotype of invasive pneumococcal disease †† & $100 \%$ & Triangular (50\%, 100\%, 150\%) & $\mathrm{N} / \mathrm{A}$ & $12,13+\dagger$ \\
\hline Net indirect effect for PCV-10 and PCV-13 & $10 \%$ & Triangular (0\%, 10\%, 30\%) & $\mathrm{N} / \mathrm{A}$ & Assumedł‡ \\
\hline Discount rate health effects & $1.5 \%$ & $\mathrm{~N} / \mathrm{A}$ & $\mathrm{N} / \mathrm{A}$ & 27 \\
\hline Discount rate costs & $4 \%$ & N/A & $\mathrm{N} / \mathrm{A}$ & 27 \\
\hline \multicolumn{5}{|l|}{ 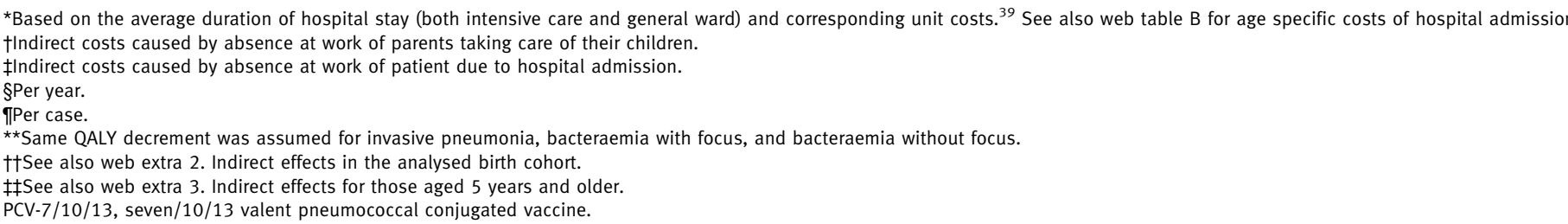 } \\
\hline
\end{tabular}




\section{Baseline disease risks}

Surveillance data on the incidence and serotype distribution of invasive pneumococcal disease before national implementation of PCV-7 were available for the period 2004-2006, including data on age, primary focus of infection, resource use, hospital admission, and outcome. ${ }^{1317}$ The case-fatality rate for meningitis and bacteraemia without focus in children was estimated to be $9 \%$ (table 1), ${ }^{17}$ which is in line with the international literature. ${ }^{5818}$ Invasive pneumonia and bacteraemia with focus were assumed not to result in death in children. ${ }^{17}$ In our model, severe mental and physical handicap resulting from meningitis was assumed to occur in 13\% of cases of pneumococcal meningitis in children, of which $50 \%$ would require special education and 25\% intensive "round the clock" institutional care. ${ }^{6}$ Jansen et al found that hearing problems occurred in $32 \%$ of cases of meningitis, of which $50 \%$ were serious enough to require a cochlear hearing device. ${ }^{17}$ Baseline risks for non-invasive pneumonia requiring hospital admission and for non-invasive pneumonia and acute otitis media treated in general practitioner surgeries were estimated from national hospital and general practitioner records, respectively (see web table $\mathrm{A}$ ).

\section{Vaccine efficacies}

Vaccine efficacy against invasive pneumococcal disease was assumed at $97.4 \%$ after two doses for all seven serotypes of pneumococcal disease covered by PCV-7 (table 1). ${ }^{19}$ This value seems to be a conservative estimate if one takes into account the fact that only one vaccine failure has been reported in the Netherlands in the first two years after introduction of routine infant vaccination in June 2006. Routine vaccination for infants in a $2+1$ dose schedule was introduced in Norway in 2006 , and similarly no vaccine failures had occurred up to June 2008. ${ }^{11}$ Protection against invasive disease was thus estimated to last for five years in the base case analysis. ${ }^{20}$ Furthermore, in randomised controlled settings, the vaccine was shown to be effective against non-invasive pneumonia and otitis media in children. ${ }^{21-23}$ For non- invasive pneumonia, efficacy of pneumococcal vaccination seems to increase with diagnostic certainty. ${ }^{23}$

In our model, we applied the efficacy estimate of $11.1 \%$ for "clinical pneumonia and perihilar findings" to children admitted to hospital with the diagnosis of pneumonia in the Netherlands. ${ }^{23}$ This definition of pneumonia seems to best fit the types of pneumonias treated in Dutch hospitals. An efficacy of $6.0 \%$ was assumed for patients who visited a general practitioner and were diagnosed with pneumonia. ${ }^{23}$ In two randomised studies, PCV-7 was found to prevent $6.4 \%$ to $7.0 \%$ of all cases of acute otitis media. ${ }^{22} 1924$ The interpretation of these studies for the Dutch setting is hampered by several factors, including the fact that the causal micro-organism is not recorded in cases of otitis media in the Netherlands. In our model, we used an overall efficacy estimate of $7.0 \%$ for otitis media on the basis of the most recent data from the Kaiser Permanente trial. ${ }^{22}$ Given evidence for the duration of protection against non-invasive pneumonia and recent US surveillance data, we assumed that vaccinated children were protected against non-invasive pneumonia and otitis media up to their second year of life, starting after the second dose of the vaccine. ${ }^{212526}$

A vaccine efficacy of $97.4 \%$ against all serotypes included was assumed for PCV-10 and PCV-13, similar to the assumed efficacy of PCV-7. In the absence of clinical data on the efficacy of PCV-10 and PCV-13 against non-invasive pneumonia and acute otitis media, the efficacy of these two vaccines was assumed to increase proportionally with the increase in serotype coverage for invasive pneumococcal disease.

\section{Indirect effects}

As well as estimations of the direct effects, we also estimated indirect effects of vaccination in our model. We included in our base case analysis herd protection against invasive pneumococcal disease for children in the birth cohort not yet fully protected by the vaccine and for non-vaccinated children, assuming this protection would be as effective as vaccination (table 2). ${ }^{12} 13$ We also increased the incidence of invasive pneumococcal disease caused by non-vaccine serotypes to

Table 2|Base case serotype coverage and efficacy for direct effects and assumptions on indirect effects for the analysed birth cohort and the remaining population (those aged 5 years or older) for PCV-7, PCV-10, and PCV-13

\begin{tabular}{|c|c|c|c|}
\hline & PCV-7 & PCV-10 & PCV-13 \\
\hline Serotypes covered & $\begin{array}{l}4,6 \mathrm{~B}, 9 \mathrm{~V}, 14 \\
18 \mathrm{C}, 19 \mathrm{~F}, 23 \mathrm{~F}\end{array}$ & $\begin{array}{l}4,6 \mathrm{~B}, 9 \mathrm{~V}, 14,18 \mathrm{C} \\
19 \mathrm{~F}, 23 \mathrm{~F}, 1,5,7 \mathrm{~F}\end{array}$ & $\begin{array}{c}\text { 4, 6B, 9V, 14, 18C, } \\
19 \mathrm{~F}, 23 \mathrm{~F}, 3,6 \mathrm{~A}, 19 \mathrm{~A}, 1,5,7 \mathrm{~F}\end{array}$ \\
\hline $\begin{array}{l}\text { Increase in invasive pneumococcal disease caused by non-vaccine serotypes in the analysed } \\
\text { birth cohort (serotype replacement) }\end{array}$ & $100 \%$ & $100 \%$ & $100 \%$ \\
\hline $\begin{array}{l}\text { Efficacy and level of herd protection against vaccine serotypes of invasive pneumococcal disease } \\
\text { in the analysed birth cohort* }\end{array}$ & $97.4 \%$ & $97.4 \%$ & $97.4 \%$ \\
\hline Net indirect effect in the remaining population $†$ & $0 \%$ & $10 \%$ & $10 \%$ \\
\hline
\end{tabular}

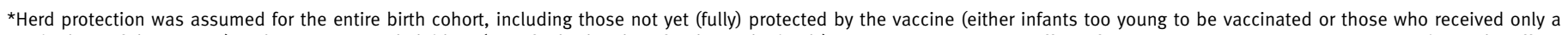

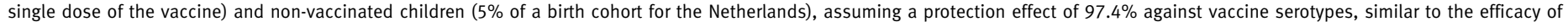
the vaccine.

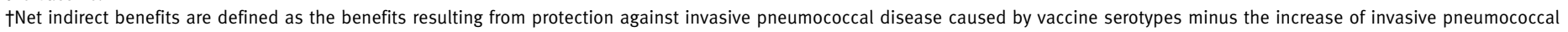

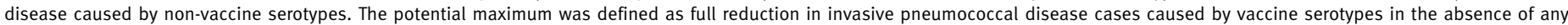

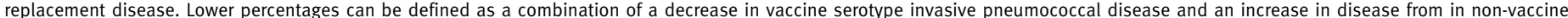
serotypes.

PCV-7/10/13, seven/10/13 valent pneumococcal conjugated vaccine. 
Table 3|Base case analysis results for the analysed Dutch birth cohort

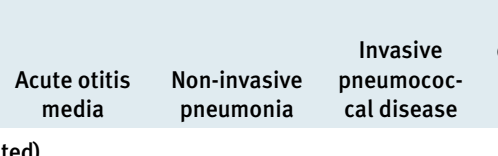

Invasive pneumococcal

disease related to net indirect

effects for individuals aged

5 years or older*

Total

Cases (undiscounted)

\begin{tabular}{lccccc}
\hline No vaccination & 170788 & 19385 & 188 & 2410 & NA \\
\hline PCV-7 & 165416 & 18979 & 117 & 210 & NA \\
\hline PCV-10 & 164664 & 18922 & 80 & 2260 & NA \\
\hline PCV-13 & 163912 & 18865 & 38 & 2229 & NA \\
\hline Cases averted & & & & & NA \\
\hline PCV-7 & 5372 & 406 & 71 & 0 & NA \\
\hline PCV-10 & 6124 & 463 & 108 & 150 & NA
\end{tabular}

\section{QALYs gained (years)}

\begin{tabular}{llllll}
\hline PCV-7 & 27 & 2 & 248 & 0 & 277 \\
\hline PCV-10 & 30 & 2 & 361 & 314 & 707 \\
\hline PCV-13 & 34 & 2 & 470 & 384 & 891 \\
\hline
\end{tabular}

Life years gained (years)

\begin{tabular}{llllcl}
\hline PCV-7 & 0 & 0 & 173 & 0 & 173 \\
\hline PCV-10 & 0 & 0 & 255 & 312 & 566 \\
\hline PCV-13 & 0 & 0 & 336 & 381 & 717
\end{tabular}

Direct savings ( $€ 1000 s)$, excluding vaccination costs

\begin{tabular}{llllll}
\hline PCV-7 & 126 & 375 & 1725 & 0 & 2226 \\
\hline PCV-10 & 144 & 427 & 2454 & 1398 & 4422 \\
\hline PCV-13 & 161 & 479 & 3181 & 1696 & 5518 \\
\hline
\end{tabular}

Indirect savings (€1000s; direct effects) related to production losses

\begin{tabular}{llllll}
\hline PCV-7 & 320 & 74 & 46 & 0 & 440 \\
\hline PCV-10 & 365 & 84 & 67 & 161 & 677 \\
\hline PCV-13 & 410 & 94 & 93 & 202 & 799
\end{tabular}

*Only net indirect effects against invasive pneumococcal disease were included in the model for individuals aged 5 years or older. For PCV-7, no net indirect effects were included into the model for individuals aged 5 years or older in the base case analysis.

NA, not applicable; PCV-7/10/13, seven/10/13 valent pneumococcal conjugated vaccine; QALY, quality adjusted life years.
$100 \%$ for the analysed birth cohort (that is, we doubled the incidence of invasive pneumococcal disease caused by non-vaccine serotypes) on the basis of surveillance data from early after national introduction of PCV-7 in the Netherlands and the UK. ${ }^{12}{ }^{13}$ See web extra 2 for a more in depth description of the assumptions for our estimation of indirect effects in the birth cohort.

No serotype information for acute otitis media and non-invasive pneumonia is available in the Netherlands, and serotype replacement for these diseases may be assumed to be already included in the vaccine efficacy estimates in the first efficacy studies. ${ }^{21-23}$ Therefore, we did not include an additional increase of nonvaccine serotype disease but also left out potential herd effects for otitis media and non-invasive pneumonia (see web extra 2).

We assumed in our base case analysis for PCV-7 that no net indirect effect would exist for individuals outside the modelled cohort. This assumption was made because no reduction in the incidence of invasive pneumococcal disease has been observed after the introduction of routine vaccination with PCV-7 for individuals 5 years of age or older and because the observed herd protection effect in the UK in the third year after introducing routine vaccination was completely countered by a rise in invasive pneumococcal disease caused by non-vaccine serotypes. ${ }^{12}$ In this respect, net indirect effects are defined as cases of invasive disease averted by herd protection minus invasive cases of replacement disease.

Net indirect effects may occur in the future, especially if serotype coverage is extended by a change from seven serotype vaccines to vaccines with broader serotype coverage. ${ }^{1317}$ Therefore, in the base case analysis for PCV-10 and PCV-13, a net indirect effect for invasive disease at $10 \%$ of the potential maximum was applied for those aged 5 years or older (see web extra 3 ). In particular, the potential maximum was defined as prevention of all cases of invasive disease caused by serotypes in the vaccine and absence of any replacement disease. Net protective indirect benefits against otitis media and non-invasive pneumonia were not included in any of the analyses. ${ }^{25}$

Given that there is much uncertainty about the development of indirect effects, these assumptions were varied over a wide range in the sensitivity analyses.

\section{Outcome measures and cost effectiveness analysis}

The simulation model tracks all the specific disease cases and the deaths, costs, changes in QALYs and life years, and indirect effects (herd protection and serotype replacement). We were able to determine the net costs and net life years and QALYs gained by summing all the costs, life years, and QALYs and calculating the differences for the evaluations with and without vaccination. The incremental cost effectiveness ratio was calculated by dividing the net costs by either life years or QALYs. Health effects and cost were discounted at $1.5 \%$ and $4 \%$ for time preference, respectively, according to the Dutch guidelines for cost effectiveness research. ${ }^{27}$

Incremental cost effectiveness ratios for routine vaccination were calculated by comparing different vaccination schedules against no vaccination. Following recently published evidence on the efficacy of PCV-7 in reduced dose schedules, ${ }^{2829}$ we investigated the effect of a three dose schedule (that is, $2+1$ ) to test the effect of lower total vaccination costs (see web extra 4). We also forecasted the incremental cost effectiveness of potential shifts from PCV-7 to pneumococcal vaccines that include additional serotypes (that is, PCV-10 and PCV-13).

For PCV-7, the estimated current cost of $€ 50$ per dose within the Dutch national immunisation programme was used. ${ }^{616}$ For PCV-13, the officially listed price of $€ 68.56$ was applied, with administration costs of $€ 5.95$ being added (total cost per dose $€ 74.51) .{ }^{16}$ For PCV-10, no officially listed price is available in the Netherlands. Given that we know the pricing of PCV-10 in other countries is conservative compared with PCV-13, we assumed the total cost per dose of PCV-10 at the midpoint between PCV-7 and PCV-13 (that is, €62.25). ${ }^{30}$ 


\section{Scenario and sensitivity analyses}

We performed univariate, threshold, scenario, and probabilistic sensitivity analyses. In the univariate sensitivity analyses, all relevant parameters were varied by $25 \%$ to explore the impact of each parameter relative to each other. One specific threshold analysis was performed in which the effect of the parameter on the incremental cost effectiveness ratio was investigated by varying the net indirect effects on individuals aged 5 years or older over a range of $0 \%$ to $30 \%$. For the probabilistic sensitivity analyses, parameters were generated using Monte Carlo sampling, with outcome values generated by running the model 5000 times. Log normal, beta, and triangular distributions were used except for multinomial probabilities, where Dirichlet distributions were assumed (see table 1 for specific distributions).

\section{RESULTS}

\section{Cost effectiveness of PCV-7}

In the base case analysis, the estimated burden of pneumococcal infection for a birth cohort followed for five years was 170788 cases of acute otitis media and 19385 cases of non-invasive pneumonia, of which 2645 cases would result in hospital admission (table 3 ). Applying the base case assumptions, 5372 (31\%) cases of acute otitis media and $406(21 \%)$ cases of noninvasive pneumonia would be prevented by vaccination with PCV-7, corresponding to gains of 27 and 2 QALYs, respectively.

Additionally, 188 cases of invasive pneumococcal disease a year were estimated in children under 5 years of age: 65 cases of meningitis; 45 cases of invasive pneumococcal disease; 38 cases of bacteraemia with focus; and 40 cases of bacteraemia without focus. In total, $71(38 \%)$ cases of invasive disease would be prevented by vaccination with PCV-7, corresponding to a total gain of 173 life years or 248 QALYs.

In addition to the health gains, vaccination with PCV-7 would also prevent approximately $€ 2.2$ million of direct costs and $€ 0.4$ million of indirect costs. Assuming a four dose schedule, the annual cost of vaccination is estimated at $€ 34.2$ million. Dividing the incremental costs by the incremental health benefits results in an incremental cost effectiveness ratio of $€ 113891$ per QALY gained for PCV-7. An incremental cost effectiveness ratio of less than $€ 50000$ per QALY would be required for PCV-7 to be regarded as potentially cost effective. Shifting from a $3+1$ dose schedule to a $2+1$ regimen could improve cost effectiveness of PCV-7 to $€ 82975$ per QALY (table 4).

\section{Cost effectiveness of PCV-10 and PCV-13}

Compared with no vaccination, vaccination with PCV-10 would prevent 6124 cases of otitis media, 463 cases of non-invasive pneumonia, and 258 cases of invasive pneumococcal disease, of which 150 would be averted by net indirect effects in individuals aged 5 years and older. Overall these health benefits would result in a gain of 707 QALYs. Vaccination with PCV-13 would prevent 6876 cases of otitis media, 520 cases of non-invasive pneumonia, and 331 cases of invasive pneumococcal disease, resulting in a total gain of 891 QALYs.

Dividing the incremental costs by the incremental health benefits for the 10 valent and 13 valent vaccines produced incremental cost effectiveness ratios of $€ 52947$ and $€ 50042$ per QALY for PCV-10 and PCV-13, respectively. A $2+1$ dose schedule could reduce these incremental cost effectiveness ratios to $€ 37891$ for PCV-10 and to €35 743 for PCV-13 (table 4). A $25 \%$ reduction in the vaccine price of PCV-10 and PCV-13 (to $€ 50$ per dose, the cost of

Table 4 Incremental cost effectiveness ratios in the base case analysis, sensitivity analysis, and several scenario analyses

\begin{tabular}{|c|c|c|c|}
\hline & PCV-7 (€/QALY) & PCV-10 (€/QALY) & PCV-13 (€/QALY) \\
\hline \multicolumn{4}{|l|}{$3+1$ dose schedule } \\
\hline Without net positive indirect effects for individuals aged 5 years or older* & $113891 \dagger$ & 99151 & 91705 \\
\hline With $10 \%$ net positive indirect effects for individuals aged 5 years or older* & 59937 & $52947 \dagger$ & $50042 \dagger$ \\
\hline With $20 \%$ net positive indirect effects for individuals aged 5 years or older* & 39698 & 35146 & 33479 \\
\hline \multicolumn{4}{|l|}{$2+1$ dose schedule } \\
\hline Without net positive indirect effects for individuals aged 5 years or older* & 82975 & 72083 & 66572 \\
\hline With $10 \%$ net positive indirect effects for individuals aged 5 years or older* & 43070 & 37891 & 35743 \\
\hline With $20 \%$ net positive indirect effects for individuals aged 5 years or older* & 28101 & 24718 & 23488 \\
\hline Reduction in the cost of the vaccine ( $€ 50$ per dose) $\ddagger$ & NA† & 41106 & 31250 \\
\hline Excluding herd effects in the analysed birth cohort for invasive pneumococcal disease & 129069 & 57770 & 55055 \\
\hline Including herd effects in the analysed birth cohort for non-invasive pneumococcal diseasef & 111153 & 52211 & 49407 \\
\hline Higher utility losses $\ddagger \S$ & 67581 & 40136 & 38664 \\
\hline Exclusion of productivity losses (analysis from a healthcare perspective) $\ddagger$ & 115481 & 53904 & 50938 \\
\hline Efficacy against acute otitis mediał $\mathbb{\prod}$ & 78527 & 43048 & 41457 \\
\hline
\end{tabular}

*Inclusion of net positive indirect effects (herd protection against vaccine serotype disease minus increases in non-vaccine serotype pneumococcal disease). See also web extra 2. †Base case scenario.

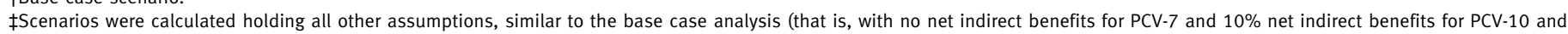
P(V-13).

SUtilities reported by Prosser et al were used for children aged up to 5 years old ${ }^{40}$

IEfficacy against acute otitis media was assumed to be $33.6 \%$, as was shown for the precursor vaccine of PCV-10 by Prymula et al. ${ }^{41}$

$\mathrm{NA}$, not applicable; PCV-7/10/13, seven/10/13 valent pneumococcal conjugated vaccine; QALY, quality adjusted life year. 


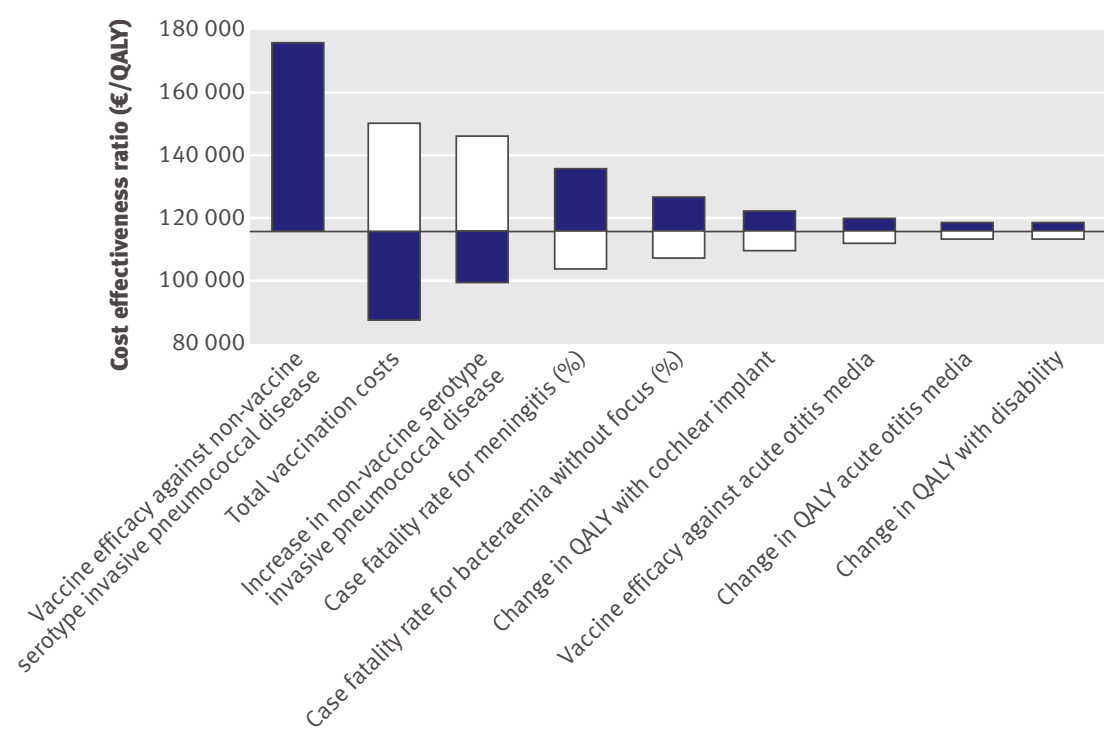

Fig 2 | Sensitivity analysis on the base case cost effectiveness ratio for the seven valent pneumococcal conjugated vaccine. The parameters were varied by $25 \%$. Dark bars show the incremental cost effectiveness ratio after a $25 \%$ decrease in the parameter, whereas light bars show the incremental cost effectiveness ratio after a $25 \%$ increase (note that it was not possible to increase vaccine efficacy). QALY, quality adjusted life year

PCV-7) would reduce the cost effectiveness ratios to $€ 41106$ and $€ 31250$, respectively. Assuming both a dose (to three doses) and a price reduction (to $€ 50$ per dose), the cost effectiveness ratios for PCV-10 and PCV-13 would be as low as $€ 29013$ and $€ 21654$ per QALY, respectively

\section{Scenario and sensitivity analyses}

Figure 2 shows the parameters that produced the largest variation in the cost effectiveness ratio for PCV-7 when varied by $25 \%$. Apart from vaccine efficacy against invasive pneumococcal disease, the most important determinants of the cost effectiveness of PCV were the total vaccination costs, the increase in invasive pneumococcal disease caused by non-vaccine serotypes, and the case fatality rate for meningitis. In univariate sensitivity analyses for PCV-10 and PCV13 , generally similar but smaller changes in the incremental cost effectiveness ratio were observed. The changes were smaller because of the relative importance of indirect benefits in the unvaccinated population for PCV-10 and PCV-13.

Figure 3 shows the impact of varying the level of net indirect effects of vaccination in individuals aged 5 years or over. At least $14 \%$ of the estimated net indirect effect would be needed in order to make PCV-7 cost effective (that is, less than $€ 50000$ per QALY). Several scenario analyses are displayed in table 4, which again show the large impact of indirect effects and reduced dose schedules on the cost effectiveness of pneumococcal vaccination.

Finally, figure 4 shows cost effectiveness acceptability curves for six different scenarios. This figure clearly shows that administering PCV -7 in a $3+1$ dose schedule cannot be considered as cost effective compared with no vaccination. The incremental cost effectiveness ratios of PCV-10 and PCV-13 are likely to be more favourable than that for PCV-7, yet still the total costs of vaccination should be reduced in order to unambiguously consider vaccination cost effective.

\section{DISCUSSION}

Our economic analysis indicates that the current national vaccination programme with PCV-7 in the Netherlands is not cost effective. As several papers suggest that lowering the number of doses from four to three will not affect the vaccine efficacy for the pneumococcal vaccine, ${ }^{11282931}$ we investigated the potential impact of such reduced-dose schedules. Although a 2 +1 reduced dose schedule could lower the total cost of vaccination and, therefore, reduce the incremental cost effectiveness ratio by approximately $30 \%$, it is unlikely that universal vaccination with PCV-7 will become acceptable on the grounds of cost effectiveness.

More favourable incremental cost effectiveness ratios were shown for PCV-10 and PCV-13, as long as net positive indirect effects for individuals aged 5 years or older were included in the analyses. In particular, scenarios that used reduced total vaccination costs by using a $2+1$ dose schedule showed that incremental cost effectiveness ratios would decrease down to $€ 37891$ and $€ 35743$ per QALY for PCV-10 and PCV-13, respectively. These ratios are likely to be considered as cost effective given various country specific thresholds.

\section{Strengths and weaknesses}

This is the first economic evaluation of national vaccination against pneumococcal disease that has included serotype replacement for the analysed birth cohort by using post-vaccination data. ${ }^{12} 13$ We estimated the number of cases of invasive pneumococcal disease averted by vaccination and the increase in invasive pneumococcal disease caused by non-vaccine serotypes on the basis of the most recent data available. ${ }^{17}$ Given the relatively small number of cases reported during the surveillance period of two years, our predictions regarding the increase of disease caused by nonvaccine serotypes may have limited precision; however, they are based on the best data currently available. In particular, the estimated increase of $100 \%$ for invasive disease caused by serotypes not covered by PCV-7 was based on national observational studies from the Netherlands and the UK..$^{1217}$ On the one hand, this specific assumption may be too pessimistic. On the other hand, data from the UK show an ongoing increase in the cases of invasive pneumococcal disease caused by non-vaccine serotypes and no plateau has yet been reached in the third year after PCV-7 introduction, suggesting that the eventual increase in disease caused by non-vaccine serotypes might even be higher. ${ }^{12}$ There are, however, some important differences between the Netherlands and the UK. In contrast to the Netherlands, the UK uses a reduced dose schedule of PCV-7 at 2, 4, and 13 months. Also, the introduction of PCV-7 in the UK was followed by a catch-up 
programme for all children aged less than 2 years. In the Netherlands, by contrast, vaccination was implemented without a catch-up programme. Several alternative scenarios regarding serotype replacement were explored in the sensitivity analyses, which showed that our conclusions regarding the incremental cost effectiveness ratios for all three vaccines were quite robust.

In our base case analysis for PCV-7, we assumed that there was no net indirect effect of vaccination for individuals outside the modelled birth cohort because no overall reduction in invasive pneumococcal disease in non-vaccinees has been observed in any European country, in contrast to the US. ${ }^{121315}$ The difference between results obtained in the US and those recorded in Europe may be partly explained by the $60 \%$ to $70 \%$ coverage of the seven vaccine serotypes in Europe, compared with the more than $80 \%$ coverage in the US. ${ }^{32}$ This disparity leaves more room for replacement disease in Europe. Country specific differences in the circulating serotypes causing disease (inclusive of secular changes in time) could also contribute to the lower overall reduction of invasive pneumococcal disease in Europe compared with the US. ${ }^{33}$ Furthermore, in the Netherlands, as in most parts of Europe, the baseline incidence rates of invasive pneumococcal disease in children are substantially lower than in the US and almost exclusively based on culture confirmed cases of children admitted to hospital. ${ }^{1734}$ Another potentially relevant difference in the introduction of PCV-7 in the Netherlands compared with the US is the high vaccine uptake $(>95 \%)$ among all newborns in the Netherlands for all four doses of the vaccination, which could potentially lead to more rapid development of replacement disease. ${ }^{34}$

Potential net indirect effects in non-vaccinees were modelled using straightforward calculus. Ideally, the impact of pneumococcal vaccination should have been modelled using a so called dynamic transmission model, in which the transmission and carriage of Streptococcus pneumoniae is taken explicitly into account. However, because the transition dynamics of $S$ pneumoniae are complex and serotype dependent, and

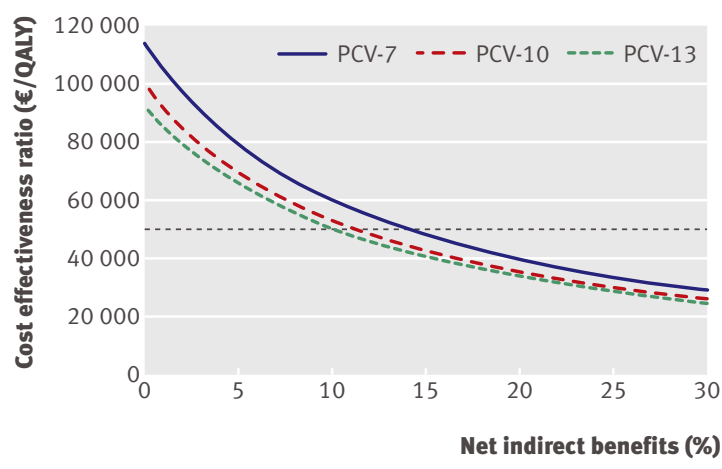

Fig 3 The effect on cost effectiveness ratios of varying the level of net indirect effect of vaccination for individuals aged 5 years or older. The horizontal dashed line shows the threshold at $€ 50000$ per QALY. PCV-7/10/13, seven/10/13 valent pneumococcal conjugated vaccine $(3+1$ dose schedule); QALY, quality adjusted life year detailed data regarding these transmission dynamics are also quite limited, dynamically modelling all relevant serotypes of $S$ pneumoniae would be very complicated. For PCV-10 and PCV-13, a net indirect effect of $10 \%$ was included in the base case analysis. This estimate of indirect benefit may be conservative if compared with the much higher net indirect protective benefits observed in the US after implementation of routine vaccination with similar or lower vaccine serotype coverage. ${ }^{2-4}$

Furthermore, we did not include the benefits arising from the prevention of antibiotic resistance in our model because the impact of this inclusion is expected to be small given that penicillin resistance is less than $0.4 \%$ in the Netherlands. ${ }^{17}$ Finally, similar to almost all previous cost effectiveness analyses for pneumococcal vaccination, our analytic time frame was equal to the assumed protection period, after which we assumed that health effects and costs would be similar in the vaccinated and unvaccinated group.

\section{Comparison with other studies}

The cost effectiveness of PCV-7 is worse than that calculated in our previous studies and in other recent health economic studies. ${ }^{5-10161835}$ This disparity is mostly because of the exclusion of herd protection effects and the inclusion of serotype replacement in our study. Other factors contributing to the worse incremental cost effectiveness ratio were the use of a lower death rate for invasive pneumococcal disease and lower indirect costs than in our previous studies. ${ }^{6} 16$

Several recently published cost effectiveness studies included net vaccine benefits for unvaccinated adults and elderly people in their base case analysis. ${ }^{5-7935}$ These studies reported vaccination to be cost saving ${ }^{59}$ or at least cost effective. ${ }^{6735}$ The three studies that excluded herd protection in the base case analysis reported relatively unfavourable cost effectiveness ratios for PCV-7 compared with other recommended infant vaccinations. ${ }^{81618}$ When we excluded the increase in invasive pneumococcal disease caused by non-vaccine serotypes but left all other assumptions the same as in the base case analysis, our results were similar to those of these three studies - that is, we found an unfavourable cost effectiveness ratio. ${ }^{81618}$

Our cost effectiveness results show that the current vaccination schedule for PCV-7 might be far more expensive per QALY gained compared with other routine infant vaccination programmes recently implemented, such as for human papilloma virus ${ }^{36}$, or with other vaccines that have not yet been implemented in a national programme in the Netherlands, such as hepatitis $\mathrm{B}^{37}$ and varicella. ${ }^{38}$

\section{Implications and future research}

Administration of PCV-7 at 2, 3, 4, and 11 months was introduced to the Netherlands as part of the national immunisation programme in 2006 partially on the basis of favourable cost effectiveness data. The current analysis shows unfavourable cost effectiveness of the 


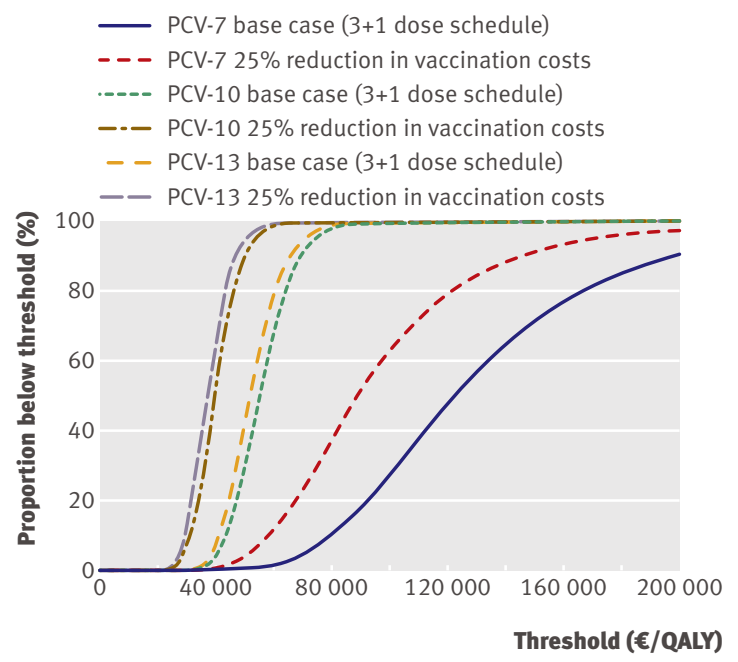

Fig $4 \mid$ Cost effectiveness acceptability curves for base case vaccination schedules and for alternative scenarios for PCV-7, PCV-10, and PCV-13

PCV-7 3+1 dose schedule because of increases in invasive disease caused by non-vaccine serotypes, which offset the herd protective benefits in individuals outside the analysed birth cohort. Although the cost effectiveness of PCV-7 is unfavourable from a health economics point of view, it is favourable from a public health point of view - a significant decrease in cases of pneumococcal disease has occurred in the Netherlands over the past two years. ${ }^{13}$ Switching to the 10 valent or 13 valent vaccine would extend the serotype coverage to a higher level than that currently achieved with PCV-7, which might reduce the potential for disease caused by non-vaccine serotypes and increase the overall benefits in vaccinated children.

Herd protective effects are more likely to occur with broad vaccine coverage, rendering vaccination potentially cost effective. Vaccination would be particularly cost effective if a more valent vaccine is used in combination with dose reductions, price reductions, or both Our paper should help guide future decisions to

\section{WHAT IS ALREADY KNOWN ON THIS TOPIC}

Recent pneumococcal surveillance studies show a significant increase in non-vaccine serotype disease, which reduces the overall health effects of vaccination and offsets potential positive herd protection benefits in unvaccinated individuals

Existing economic analyses of pneumococcal vaccination did not incorporate the increase in disease caused by non-vaccine serotypes, and most included too optimistic herd protective effects

\section{WHAT THIS STUDY ADDS}

The current four dose $(3+1)$ schedule of the seven valent pneumococcal conjugated vaccine used in the Netherlands is not cost effective

Vaccination with 10 valent or 13 valent vaccines could substantially reduce the incremental cost effectiveness of pneumococcal vaccination to a potentially acceptable level

Reducing the total programme cost for pneumococcal vaccination by reducing dose schedules, reducing vaccine prices, or both is necessary to unambiguously render routine infant vaccination cost effective in the Netherlands potentially reduce doses of pneumococcal vaccine or to shift from PCV-7 to vaccines that cover additional serotypes. Further research should be directed to building a dynamic model to entangle and explicitly predict the indirect effects of disease replacement and herd protection on vaccine efficacy and thus further enhance the validity of cost effectiveness approaches applied to pneumococcal vaccination.

Contributors: MJP, EH, and GvdD designed the study. MHR, AJvH, and MJP designed the computer model and carried out the computer simulations and analysis. Data analyses were performed by AGSCJ, GDR, and MHR under supervision of EAMS, MJP, and AvdE. MHR, MJP, AJvH, and EAMS drafted the manuscript. All authors commented on drafts and contributed to the final version. MHR and MJP are the guarantors of the study. Funding: MHR was funded by an unrestricted grant from Wyeth Hoofddorp. AJvH was financed by the Netherlands Vaccine Institute, Bilthoven. This work has been previously presented at a workshop on pneumococcal vaccines at the European Public Health Association conference in Lisbon, Portugal, which was supported by a research grant from GlaxoSmithKline Netherlands. The authors' work was independent of the funders, who had no role in the study design, analysis of data, writing of the manuscript, or decision to submit for publication. Competing interests: All authors have completed the Unified Competing Interest form at www.icmje.org/coi_disclosure.pdf (available on request from the corresponding author) and declare: (1) MHR was funded by an unrestricted grant from Wyeth Hoofddorp; and AJvH was financed by the Netherlands Vaccine Institute, Bilthoven; (2) MJP has received travel grants from GlaxoSmithKline and Wyeth to attend expert meetings in Reykjavik, Iceland, and Istanbul, Turkey; EAMS has received unrestricted grants from Wyeth and Baxter for research, consulting fees from Wyeth and GlaxoSmithKline, lecturing fees from Wyeth, and grant support from Wyeth and GlaxoSmithKline for vaccine studies; and AvdE has received unrestricted grants from Wyeth and Novartis; (3) No spouses, partners, or children with relationships with commercial entities that might have an interest in the submitted work; (4) No non-financial interests that may be relevant to the submitted work.

Data sharing: No additional data available.

1 Health Council of the Netherlands. Vaccination of infants against pneumococcal infections [in Dutch]. 2005. www.gezondheidsraad. nl/sites/default/files/05@13N.pdf.

2 Hsu HE, Shutt KA, Moore MR, Beall BW, Bennett NM, Craig AS, et al. Effect of pneumococcal conjugate vaccine on pneumococcal meningitis. N Engl J Med 2009;360:244-56.

3 Lexau CA, Lynfield R, Danila R, Pilishvili T, Facklam R, Farley MM, et al. Changing epidemiology of invasive pneumococcal disease among older adults in the era of pediatric pneumococcal conjugate vaccine. JAMA 2005;294:2043-51.

4 Whitney CG, Farley MM, Hadler J, Harrison LH, Bennett NM, Lynfield R, et al. Decline in invasive pneumococcal disease after the introduction of protein-polysaccharide conjugate vaccine. $N$ EnglJ Med 2003:348:1737-46.

5 Claes C, Reinert RR, von der Schulenburg JM. Cost effectiveness analysis of heptavalent pneumococcal conjugate vaccine in Germany considering herd immunity effects. Eur J Health Econ 2009;10:25-38.

6 Hubben GA, Bos JM, Glynn DM, van der Ende A, van Alphen L, Postma MJ. Enhanced decision support for policy makers using a web interface to health-economic models-illustrated with a costeffectiveness analysis of nation-wide infant vaccination with the 7valent pneumococcal conjugate vaccine in the Netherlands. Vaccine 2007;25:3669-78.

7 McIntosh ED, Conway P, Willingham J, Hollingsworth R, Lloyd A Pneumococcal pneumonia in the UK-how herd immunity affects the cost-effectiveness of 7-valent pneumococcal conjugate vaccine (PCV). Vaccine 2005;23:1739-45.

8 Melegaro A, Edmunds WJ. Cost-effectiveness analysis of pneumococcal conjugate vaccination in England and Wales. Vaccine 2004;22:4203-14.

9 Silfverdal SA, Berg S, Hemlin C, Jokinen I. The cost-burden of paediatric pneumococcal disease in Sweden and the potential costeffectiveness of prevention using 7-valent pneumococcal vaccine. Vaccine 2009;27:1601-8.

10 Tilson L, Usher C, Butler K, Fitzsimons J, O’Hare F, Cotter S, et al. Economic evaluation of a universal childhood pneumococcal conjugate vaccination strategy in Ireland. Value Health 2008;11:898-903. 
11 Vestrheim DF, Lovoll O, Aaberge IS, Caugnant DA, Holby EA, Bakke H, et al. Effectiveness of a $2+1$ dose schedule pneumococcal conjugate vaccination programme on invasive pneumococcal disease among children in Norway. Vaccine 2008;26:3277-81.

12 Health Protection Agency. Cumulative weekly number of reports of invasive pneumococcal disease in England and Wales by epidemiological year July-June. 2009. www.hpa.org.uk.

13 Rodenburg GD, de Greeff SC, Jansen AG, de Melker HE, Schouls LM, Hak E, et al. Effects of pneumococcal conjugate vaccine 2 years after its introduction, the Netherlands. Emerg Infect Dis 2010;16:816-23.

14 Munoz-Almagro C, Jordan I, Gene A, Latorre C, Garcia-Garcia JJ, Pallares R. Emergence of invasive pneumococcal disease caused by nonvaccine serotypes in the era of 7-valent conjugate vaccine. Clin Infect Dis 2008;46:174-82.

15 Lepoutre A, Varon E, Georges S, Gutmann L, Levy-Bruhl D. Impact of infant pneumococcal vaccination on invasive pneumococcal diseases in France, 2001-2006. Euro Surveill 2008;13:ii,18962.

16 Bos JM, Rumke H, Welte R, Postma MJ. Epidemiologic impact and cost-effectiveness of universal infant vaccination with a 7-valent conjugated pneumococcal vaccine in the Netherlands. Clin Ther 2003;25:2614-30

17 Jansen AGSC, Rodenburg GD, Greef SC, Hak E, Veenhoven RH, Spanjaard L, et al. Invasive pneumococcal disease in the Netherlands: syndromes, outcome and potential vaccine benefits. Vaccine 2009;27:2394-401.

18 Giorgi-Rossi P, Merito M, Borgia P. Cost-effectiveness of introducing the conjugated pneumococcal vaccine to routine free immunizations for infants in Lazio, Italy. Health Policy 2009;89:225-38.

19 Black S, Shinefield H, Fireman B, Lewis E, Ray P, Hansen JR, et al. Efficacy, safety and immunogenicity of heptavalent pneumococcal conjugate vaccine in children. Northern California Kaiser Permanente Vaccine Study Center Group. Pediatr Infect Dis / 2000;19:187-95.

20 Madhi SA, Adrian P, Kuwanda L, Jassat W, Jones S, Little T, et al. Long term immunogenicity and efficacy of a 9-valent conjugate pneumococcal vaccine in human immunodeficient virus infected and non-infected children in the absence of a booster dose of vaccine. Vaccine 2007;25:2451-7.

21 Hansen J, Black S, Shinefield H, Cherian T, Benson J, Fireman B, et al. Effectiveness of heptavalent pneumococcal conjugate vaccine in children younger than 5 years of age for prevention of pneumonia: updated analysis using World Health Organization standardized interpretation of chest radiographs. Pediatr Infect Dis I 2006;25:779-81.

22 Fireman B, Black SB, Shinefield HR, Lee J, Lewis E, Ray P. Impact of the pneumococcal conjugate vaccine on otitis media. Pediatr Infect Dis / 2003;22:10-6

23 Black SB, Shinefield HR, Ling S, Hansen J, Fireman B, Spring D, et al. Effectiveness of heptavalent pneumococcal conjugate vaccine in children younger than five years of age for prevention of pneumonia. Pediatr Infect Dis J 2002;21:810-5.

24 Eskola J, Kilpi T, Palmu A, Jokinen J, Haapakoski J, Herva E, et al. Efficacy of a pneumococcal conjugate vaccine against acute otitis media. N Engl J Med 2001;344:403-9.

25 Nelson JC, Jackson M, Yu O, Whitney CG, Bounds L, Bittner R, et al. Impact of the introduction of pneumococcal conjugate vaccine on rates of community acquired pneumonia in children and adults. Vaccine 2008;26:4947-54.

26 Centers for Disease Control and Prevention. Pneumonia hospitalizations among young children before and after introduction of pneumococcal conjugate vaccine-United States, 1997-2006. MMWR Morb Mortal Wkly Rep 2009;58:1-4.

27 Brouwer WB, Niessen LW, Postma MJ, Rutten FF. Need for differentia discounting of costs and health effects in cost effectiveness analyses. BMJ 2005;331:446-8.

28 Goldblatt D, Southern J, Ashton L, Richmond P, Burbidge P, Tasevska J, et al. Immunogenicity and boosting after a reduced number of doses of a pneumococcal conjugate vaccine in infants and toddlers. Pediatr Infect Dis / 2006;25:312-9.

29 Whitney CG, Pilishvili T, Farley MM, Schaffner W, Craig AS, Lynfield R, et al. Effectiveness of seven-valent pneumococcal conjugate vaccine against invasive pneumococcal disease: a matched case-control study. Lancet 2006;368:1495-502.

30 Vespa G, Constenla DO, Pepe C, Safadi MA, Berezin E, Cassio de Moraes J, et al. Estimating the cost-effectiveness of pneumococcal conjugate vaccination in Brazil. Rev Panam Salud Publica 2009;26:518-28.

31 Kayhty H, Ahman H, Eriksson K, Sorberg M, Nilsson L. Immunogenicity and tolerability of a heptavalent pneumococcal conjugate vaccine administered at 3, 5 and 12 months of age. Pediatr Infect Dis / 2005;24:108-14.

32 Hausdorff WP, Bryant J, Paradiso PR, Siber GR. Which pneumococcal serogroups cause the most invasive disease: implications for conjugate vaccine formulation and use, part I. Clin Infect Dis 2000;30:100-21.

33 Fenoll A, Granizo JJ, Aguilar L, Gimenez MJ, Aragoneses-Fenoll L, Hanquet G, et al. Temporal trends of invasive Streptococcus pneumoniae serotypes and antimicrobial resistance patterns in Spain from 1979 to 2007. J Clin Microbiol 2009;47:1012-20.

34 Centers for Disease Control and Prevention. Invasive pneumococcal disease in children 5 years after conjugate vaccine introductioneight states, 1998-2005. MMWR Morb Mortal Wkly Rep 2008;57:144-8

35 Ray GT, Whitney CG, Fireman BH, Ciuryla V, Black SB. Costeffectiveness of pneumococcal conjugate vaccine: evidence from the first 5 years of use in the United States incorporating herd effects. Pediatr Infect Dis J 2006;25:494-501.

36 Jit M, Choi YH, Edmunds WJ. Economic evaluation of human papillomavirus vaccination in the United Kingdom. BMJ 2008;337:a769.

37 Tilson L, Thornton L, O’Flanagan D, Johnson H, Barry M. Cost effectiveness of hepatitis B vaccination strategies in Ireland: an economic evaluation. Eur J Public Health 2008;18:275-82.

38 Rozenbaum MH, van Hoek AJ, Vegter S, Postma MJ. Costeffectiveness of varicella vaccination programs: an update of the literature. Expert Rev Vaccines 2008;7:753-82.

39 Oostenbrink JB, Bouwmans CAM, Koopmanschap MA, Rutten FHH. Guidelines for costing research, methods and standardized prices fo economic evaluations in health care. Dutch Health Care Insurance Board, 2004.

40 Prosser LA, Ray GT, O’Brien M, Kleinman K, Santoli J, Lieu TA. Preferences and willingness to pay for health states prevented by pneumococcal conjugate vaccine. Pediatrics 2004;113:283-90.

41 Prymula R, Peeters P, Chrobok V, Kriz P, Novakova E, Kaliskova E, et al. Pneumococcal capsular polysaccharides conjugated to protein D for prevention of acute otitis media caused by both Streptococcus pneumoniae and non-typable Haemophilus influenzae: a randomised double-blind efficacy study. Lancet 2006;367:740-8.

Accepted: 16 February 2010 